\title{
Garlic: A Phytochemical Enriched Food
}

\author{
Muhammad Qamrosh Alam*, Shahid Mahmood, Wajiha Saeed, Modassar AN Ranjha, Bakhtawar Shafique, Tahreem \\ Jawaid and Abdullah Bin Masood \\ Institute of Food Science and Nutrition, University of Sargodha, Sargodha, Pakistan \\ *Corresponding Author: Muhammad Qamrosh Alam, Institute of Food Science and Nutrition, University of Sargodha, Sargodha, Pakistan.
}

Received: August 30, 2019; Published: September 26, 2019

\section{Abstract}

Garlic (Allium sativum L.) belongs to Liliaceae family. In ancient times, Aristotle, Pliny and Hippocrates incorporated a multiple and massive use of garlic (Allium sativum L.) in order to cure innumerable diseases. Garlic (Allium sativum L.) is one of the oldest cultured plant with various medicinal properties. Garlic (Allium sativum L.) contains 33 sulphur constituents, some other compounds and also a wide diversity of minerals i.e. Copper, Calcium, Magnesium, Selenium, Zinc, Germanium, Vitamin B1, A and C, Water and Fiber etc. Also, it involves 17 amino acids i.e. lysine, histidine, aspartic corrosive tryptophan, isoleucine, arginine, threonine, glutamine, proline, cysteine, alanine, valine, swine, methionine, glycine, leucine and phenylalanine. Garlic (Allium sativum L.) has a potential to cure tumors, cardiovascular or coronary disorders and many other viral and bacterial illnesses. Garlic is considered as the best antihypertensive. In many countries, progressions in AIDS \HIV fatalities have resulted. Curative treatment in executioner cells action by the usage of garlic (Allium sativum L.). A scientific study protocol yields $100 \%$ positive outcomes by the utilization of garlic (Allium sativum L.) in regular diet.

Keywords: Garlic (Allium sativum L.); Allicin; Alliin; Cardiovascular; Antibacterial; Low Density Lipoprotein Cholesterol (LDL-C)

Garlic (Allium sativum L.) belongs to Liliaceae family is a cultured food intensely respected all via the world. At the start from central Asia, garlic is surely one of the soonest of cultured plants. The Egyptian healing papyrus and Ebers Codex notices garlic ( $A l$ lium sativum L.) as a conceivable solution for a wide variety of diseases. In ancient times, Aristotle, Pliny and Hippocrates incorporated a multiple and massive use of garlic (Allium sativum L.) in order to cure innumerable diseases [1]. Garlic (Allium sativum L.) is a common food which is utilize by numerous civilizations as an extract or enthusiasm.

Pakistan, South Korea, China, India, Spain and United States are the higher cultivators of garlic (Allium sativum L.). Garlic (Allium sativum L.) is indicated hostile to viral, bacterial and fungal attacks. In addition, garlic (Allium sativum L.) has a potential to combat against atherosclerotic plagues and tumors. In a cohort study of 900 individuals, with an assistance of United States Food and Drug Administration (USFDA), garlic is ranked $2^{\text {nd }}$ as the highly consumed supplement [2].

\section{Conceivably energetic chemical constituents of garlic}

Garlic (Allium sativum L.) encompasses a rich quantity of sulfur mixes that is responsible for 2 major characteristics i.e. sharp aroma and soothing effect. One of the most striking phyto-chemical in garlic (Allium sativum L.) is allicin (diallyl disulfide or diallyl thiosulfinate). Undoubtedly, alliin (S-ally cysteine sulfoxide) is also plentiful sulfur composite in garlic (Allium sativum L.) [3]. Cleav- ing, mincing and pulverizing aggravates the protein "allinase" that immediately convert alliinase into allicin that enhance the pungent odor of garlic (Allium sativum L.) [4]. Allicin is the basic thiosulfanate that could exist as much as 08 hours at room temperature and when stored as an extract or minced form. Alliin, including allyl disulfide and allyl cysteine are the other kinds of thiosulfanates. Pedrazza-Cheverri., et al. 2006 elaborated that composites of the allinase remains in control of thiosulfanate transformation progresses toward becoming inactivated underneath a $\mathrm{pH}$ of 3.49 or with reheating [4]. Within the lapse of 01-minute allinase movement will be annihilated due microwave radiation. According to this aspect the low molecular weight thiosulfinates are responsible for the restorative estimation of garlic. Besides the fact that allicin is considered as the massive cancer prevention agent and shopping compound, ponders are demonstrating that unique mixes may just count on extra grounded materials [6]. What's extra, more up to the minute investigate has portrayed some of the glacial combinations of steroidal and phenolic founding fact that tendered certain types of a kind pharmacological houses. In 2006, Lanzotti demonstrated these final mixes except the thiosulfinates which non-aromatic were and additionally heat consistent [5]. Apart from a component to the scrounger houses of garlic will not be influenced with the aid of warming [4].

\section{Health protocols of garlic}

Justifiably, garlic can be discussed to as certainly one of nature's first-rate crops with recuperating vigor. It may possibly repress 
and get rid of microbes, growths, scale back (pulse, blood cholesterol and glucose), prevent blood thickening, and contains hostile to tumors. It may well likewise aid the invulnerable framework to chase away contamination and look after wellness [7]. It might invigorate the lymphatic framework which facilitates the expulsion of waste gadgets from the physique. It's likewise viewed as a compelling melanoma prevention agent to be certain cells towards free radical harm. It may well hold several forms of tumor, coronary disorders, strokes and viral illnesses. Mansell and Reckless, in 1991 stressed that garlic by myself can give us greater than 200 irregular chemical compounds having a strong capability of protecting the human body from a massive range of sicknesses. Garlic due to its sulfur containing blends can undergo the degree of the human build-up with indemnification, by the help of unique and advance proteins [8].

\section{Treat cardio-vascular wellbeing}

Mortality rate is increasing due cardio-vascular system derangements, gradually. The major problem is thrombosis that is tremendously becoming much common. Blockade in coronary canals is considered as the biggest cause of mortality. The conduits, which give heart with blood and oxygen $\left(\mathrm{O}_{2}\right)$, grow to be steadily smaller as plaque develops after a while. Heart gets a cut of oxygen $\left(\mathrm{O}_{2}\right)$ and then heart failure occurs. The $0_{2}$ largest problems for coronary health diseases are hypertension and excessive serum low density lipoprotein cholesterol (LDL-C) stages. In order to justify the coronary sickness, Bordia in 1981 performed scientific experiment on rabbits to prove that not only atherosclerosis but lesions would also be appeared if garlic (Allium sativum L.) isn't utilized [9]. Consistent with a blood chemistry report in India, approximately 4032 cardiac patients that were equally divided into 02 congregations and among half of them were treated with garlic and another half were on placebo. Finally, the efficacy was shown in blood chemistry as there was a great difference between both the groups of sufferers [10]. Borek in 2006, scientifically publicized some of the disorders related to heart i.e. diminished homocysteine level, low and feeble pulse, blood vessel plaque and microcirculation augmentation [11].

\section{Garlic as blood pressure reducing agent}

For circulatory strain manipulate, garlic (Allium sativum L.) is considered as one of the major conforming remedy. Capraz in 2006 presented in-vitro trials to facilitate that garlic (Allium sativum L.) is enriched with sulfur mixes that is beneficial in an identified endogenous heart-defensive mechanism [12]. There was a reduction of high blood pressure by $15 \mathrm{mmHg}$ after consumption of $2399 \mathrm{mg}$ garlic that contains approximately $30 \mathrm{mg}$ of allicin [13]. It was further reported that after a successful consumption of allicin, there was a reduction of $09 \mathrm{mmHg}$ in diastolic pressure too [14].

\section{As a superlative immunity promoter}

With the passage of time and fluctuation in environmental factors, fatal diseases like AIDS and hepatitis is rapidly increasing, gradually. This alarming situation created a problematic and criti- cal agenda. Keeping into mind, the solution of this disaster became critical demographically. No glorious medications or suppositories are helpful. Innumerable cases have been reported with perfect cure using garlic, as it contains in it a major curative blend of sulfur containing unique constituents and amino acids that revolutionized a best way of cure in circulatory system ailments specially immunity stabilization [15]. One of the best curative yield having a capability to produce macrophages $\backslash$ executioner cells to invade against health hazardous diseases. People prone to catch disease are exercising the sedentary life habits such as exposure to lead cigarette smoking, inadequate nourished diet, logical stress and brew adulteration [16]. Morioka in 1993 deducted effective results that germanium, niacin, sulfur, thiamine, selenium and phosphorous are important constituent in garlic which have shown reasonable incitement values in incurable cases [17].

Individuals, routinely and recurrently using garlic (alliin) have revealed successive results in immunity boosting by enhancing phago-cytosis and immune-reactions. $600 \mathrm{mg}$ of garlic in a day for 3 months in mature individuals triggered best augmentations within the degree of phagocytosing fringe, immunity against E. coli microorganisms [7].

In China and United States, certain progressions in AIDS \HIV fatalities have resulted unbelievable and curative treatment in executioner cells action by the usage of garlic foodstuffs. Influential Antiviral motion has shown viral ailments in bone marrow transplant victims. A study protocol yield scientifically cent $\%$ outcomes by practice allicin in regular diet [18].

\section{As an ordinary blood thinner}

The massive portion of blood consists of red blood corpuscles, leucocytes and also platelets and fibrin which are considered in blood coagulation and can be a major foundation of coronary cardiac assault. Progression of fibrin can be reduced which results in lowering of many cardiac complaints, for instance. headache, nausea can easily be reduced. Garlic is considered as one of the beneficial and cheapest remedy in cardio-vascular diseases [19]. Garlic's another composite i.e. Ajoene, is observed as the best in controlling circulatory mechanism, responsible in thinning of blood. Nevertheless, at room temperature Ajoene remains normal, in dried garlic it is vague in crude state. Diet consisting garlic can paradigm the collapse of fibrin from 25 to $29 \%$ in entities [20].

\section{Best for coronary artery disease and hyperlipoproteinemia}

The capacity to rapidly lowering down the increased level of cholesterol and an effort to avoid plaque progress entirely succeeded by utilizing garlic to a suggested extent. Garlic tends to overwhelm low density lipoprotein (LDL) and oxidation extended [21]. A study based on hominoid surveys are blended with anticipates performed in the mid of 1991's shown massive successful consequences. As extra investigates had been led extra up-to-the-minute tactics to separate garlic, late investigation of 15 hypercholesterolemia sufferers assessed a fabric delivered from ripened garlic with red mold. 
Serum cholesterol levels and low-density-lipoproteins (LDL) have diminished on checked results by inoculation and treatment by garlic in a period of 2.5 months. High Density Lipoproteins (HDL) was inviolate and also the level of triglycerides diminished in hyperactive-triglyceridemic patients too [22]. After a lapse of sixty days, with the continuation of garlic supplementation, LDL and serum triglyceride had been reduced to 20,36 , and $36.8 \%$ [23].

Some negative outcomes of garlic

Breath odor has been the utmost common and unfavorable problem by using garlic and garlic foodstuffs, having a negative influence and outcome particularly when crude herb was utilized. Excessive amounts of garlic consumption cause nausea and vomiting. If ingested in a small proportion can treat these symptoms too [24]. Specific garlic sensitivity has been attributed to the allinase protein [25]. Consequently, the writing stressed in hostility to exploiting garlic in comparison with anticoagulant therapeutic. An 87 years of age man faced epidural hematoma with related platelet brokenness identified with intemperate garlic ingestion [26]

\section{Bibliography}

1. Murray Michael T and Joseph Pizzorno. "The encyclopedia of healing foods". Simon and Schuster (2010).

2. Timbo Babgaleh B., et al. "Dietary supplements in a national survey: prevalence of use and reports of adverse events". Journal of the American Dietetic Association 106.12 (2006): 1966-1974.

3. Lawson Larry D. "Garlic: a review of its medicinal effects and indicated active compounds". (1998): 176-209.

4. Pedraza-Chaverrí José., et al. "Garlic prevents hypertension induced by chronic inhibition of nitric oxide synthesis". Life Sciences 62.6 (1998): PL71-PL77.

5. Lanzotti Virginia. "The analysis of onion and garlic". Journal of Chromatography A 1112.1-2 (2006): 3-22.

6. Chung Lip Yong. "The antioxidant properties of garlic compounds: allyl cysteine, alliin, allicin, and allyl disulfide". Journal of Medicinal Food 9.2 (2006): 205-213.

7. Abdullah Tariq H., et al. "Garlic revisited: therapeutic for the major diseases of our times?". Journal of the National Medical Association 80.4 (1988): 439.

8. Mansell Peter and J P Reckless. "Garlic". BMJ: British Medical Journal 303.6799 (1991): 379.

9. Bordia A M K H. "Effect of garlic on blood lipids in patients with coronary heart disease". The American Journal of Clinical Nutrition 34.10 (1981): 2100-2103.
10. Yeh Gloria Y., et al. "Use of complementary therapies in patients with cardiovascular disease". The American journal of cardiology 98.5 (2006): 673-680.

11. Borek Carmia. "Garlic reduces dementia and heart-disease risk". The Journal of nutrition 136.3 (2006): 810S-812S.

12. Capraz Mustafa., et al. "Garlic, hypertension and patient education". International journal of cardiology 121.1 (2007): 130131.

13. McMahon F Gilbert and Ramon Vargas. "Can garlic lower blood pressure? A pilot study". Pharmacotherapy: The Journal of Human Pharmacology and Drug Therapy 13.4 (1993): 406-407.

14. Silagy Christopher A and H A Neil. "A meta-analysis of the effect of garlic on blood pressure". (1994): 463-468.

15. Lau Benjamin HS., et al. "Effect of an odor-modified garlic preparation on blood lipids". Nutrition Research 7.2 (1987): 139-149.

16. Salman Hertzel., et al. "Effect of a garlic derivative (alliin) on peripheral blood cell immune responses". International journal of immunopharmacology 21.9 (1999): 589-597.

17. Morioka Naoko., et al. "A protein fraction from aged garlic extract enhances cytotoxicity and proliferation of human lymphocytes mediated by interleukin- 2 and concanavalin A". Cancer Immunology, Immunotherapy 37.5 (1993): 316-322.

18. Josling Peter. "Preventing the common cold with a garlic supplement: a double-blind, placebo-controlled survey". Advances in therapy 18.4 (2001): 189-193.

19. Fukao Hideharu., et al. "Antithrombotic effects of odorless garlic powder both in vitro and in vivo". Bioscience, biotechnology, and biochemistry (2007).

20. Ernst E. "Fibrinogen: an important risk factor for atherothrombotic diseases". Annals of Medicine 26.1 (1994): 15-22.

21. Lau Benjamin HS. "Suppression of LDL oxidation by garlic compounds is a possible mechanism of cardiovascular health benefit". The Journal of Nutrition 136.3 (2006): 765S-768S.

22. Sumioka Isao., et al. "Lipid-lowering effect of monascus garlic fermented extract (MGFE) in hyperlipidemic subjects". Hiroshima Journal of Medical Sciences 55.2 (2006): 59.

23. Jeyaraj S., et al. "Effect of combined supplementation of fish oil with garlic pearls on the serum lipid profile in hypercholesterolemic subjects". Indian heart journal 57.4 (2005): 327-331. 
24. Friedman Tal., et al. "Self-inflicted garlic burns: our experience and literature review". International Journal of Dermatology 45.10 (2006): 1161-1163.

25. Kao Shao-Hsuan., et al. "Identification and immunologic characterization of an allergen, alliin lyase, from garlic (Allium sativum)". Journal of Allergy and Clinical Immunology 113.1 (2004): 161-168.

26. Saw Jun Tze., et al. "Potential drug-herb interaction with antiplatelet/anticoagulant drugs". Complementary therapies in clinical practice 12.4 (2006): 236-241.

Volume 3 Issue 10 October 2019

(c) All rights are reserved by Muhammad Qamrosh Alam., et al. 